\title{
Padrões morfológicos de diásporos de árvores e arvoretas zoocóricas no Parque Estadual de Itapuã, RS, Brasil
}

\author{
Manuela Boleman Wiesbauer ${ }^{1,4}$, Eduardo Luís Hettwer Giehl² e João André Jarenkow ${ }^{3}$
}

Recebido em 22/12/2006. Aceito em 2/07/2007

\begin{abstract}
RESUMO - (Padrões morfológicos de diásporos de árvores e arvoretas zoocóricas no Parque Estadual de Itapuã, RS, Brasil). Diásporos são as unidades de dispersão das plantas e sua morfologia está relacionada a adaptações às estratégias de dispersão e estabelecimento. Neste estudo, foram avaliadas características morfológicas de diásporos de 64 espécies de árvores e arvoretas adaptadas ao consumo por vertebrados, no Parque Estadual de Itapuã, sul do Brasil, com o objetivo de relacionar características relevantes para a dispersão, como tamanho, formato e cor dos diásporos, com aspectos relacionados ao estabelecimento de plântulas, como massa e quantidade de sementes. Foram considerados dispersos por vertebrados aqueles diásporos que apresentaram estruturas carnosas. O comprimento médio dos diásporos das espécies analisadas foi $1,7 \pm 1,67 \mathrm{~cm}(\mathrm{~N}=1.402)$. Baseando-se em um diagrama de ordenação, produzido com o comprimento e massa dos diásporos e número e massa de sementes por diásporo, as espécies foram separadas em quatro grupos morfológicos. Espécies com poucas sementes por diásporo foram associadas à dispersão por aves, mamíferos ou estratégias mistas; a presença de muitas sementes de massa elevada foi associada à síndrome de mamaliocoria; e grande número de sementes de pequena massa e tamanho, à síndrome mista. O grupo com maior riqueza (35 espécies) foi aquele com diásporos pequenos e poucas sementes, potencialmente aptos à dispersão por aves e com maior proporção em massa de sementes por diásporo.
\end{abstract}

Palavras-chave: síndrome zoocórica, dispersão de sementes, tamanho de sementes, coloração de frutos, forma de frutos

\begin{abstract}
Morphological patterns of diaspores from animal-dispersed tree and treelet species at Parque Estadual de Itapuã, Rio Grande do Sul State, Brazil). Diaspores are the dispersal units of plants and their morphology is linked to dispersal and establishment strategies. In this study we evaluated diaspore morphology in 64 tree and treelet species adapted to vertebrate consumption at Parque Estadual de Itapuã, southern Brazil. Our aim was to relate features crucial to dispersal, such as diaspore length, shape and color, to features limiting establishment success, like mass and number of seeds. We considered vertebrate-dispersed diaspores to be those with fleshy structures. Average diaspore length for the species analyzed was $1.7 \pm 1.67 \mathrm{~cm}(\mathrm{~N}=1,402)$. Based on an ordination diagram using length and mass of diaspores plus number and mass of seeds per diaspore, we separated the species into four morphological groups. Species with few seeds per diaspore were linked to bird, mammal, or mixed dispersal strategies; species with high mass and many seeds per diaspore was an indication of mammal dispersal; and a high number of small, light seeds was associated with mixed syndrome strategists. The group with highest species richness (35 species) presented small diaspores and few seeds, most of them red or black in color, potentially adapted to bird dispersal and with a greater proportion of seed mass per diaspore.
\end{abstract}

Key words: zoochoric syndrome, seed dispersal, seed size, fruit color, fruit shape

\section{Introdução}

Diásporos são as unidades de dispersão das plantas, podendo tratar-se de frutos, sementes ariladas ou infrutescências (van der Pijl 1982). A morfologia dos diásporos e das sementes está relacionada a fatores ecológicos como modo de dispersão de sementes (van der Pijl 1982; Westoby et al. 1990; Hammond \& Brown 1995; Jordano 1995), predação de sementes (Janzen 1969; Harper et al. 1971; Geritz 1998; Mack 1998) e estratégia de estabelecimento das plantas (Harper et al.
1971; Hammond \& Brown 1995), além de estar associada à morfologia da flor (Primack 1987) e à filogenia (Janson 1992; Kelly \& Purvis 1993; Jordano 1995). O tamanho das sementes também pode ser bastante variável em uma mesma espécie ou indivíduo, podendo haver variação morfológica associada ao número de sementes por diásporo (Michaels et al. 1988).

As diversas convergências morfológicas, tanto do diásporo como da própria planta, causadas por pressão seletiva dos agentes dispersores, determinaram padrões

\footnotetext{
1 Universidade Federal do Rio Grande do Sul

2 Universidade Federal do Rio Grande do Sul, Av. Bento Gonçalves, 9500, Prédio 43433, 91501-970 Porto Alegre, RS, Brasil (eduardohet@gmail.com)

3 Universidade Federal do Rio Grande do Sul, Departamento de Botânica, Av. Bento Gonçalves 9500, Prédio 43433, $91501-970$ Porto Alegre, RS, Brasil (jarenkow@portoweb.com.br)

4 Autor para correspondência: manu_wiesbauer@yahoo.com.br
} 
denominados síndromes de dispersão (van der Pijl 1982). A síndrome de zoocoria é caracterizada pelo desenvolvimento de estruturas carnosas nos diásporos, que servem de atrativo e recompensa à fauna. De acordo com o grupo de vertebrados dispersores, se pode ainda identificar algumas "subsíndromes", sendo estas a ictiocórica (peixes), saurocórica (répteis), ornitocórica (aves) e mamaliocórica (mamíferos), dentro desta distingui-se ainda quiropterocórica (morcegos) (van der Pijl 1982).

Nas angiospermas, a dispersão por aves e mamíferos tem especial relevância devido a atual abundância destes animais, que supostamente exerceram um papel decisivo na irradiação desse grupo de plantas (Tiffney 1984; Tiffney 2004). Diásporos adaptados à dispersão por aves são freqüentemente inodoros, de coloração contrastante com a vegetação, como roxa/atropurpúrea, vermelha, laranja e branca (Ridley 1930; van der Pijl 1982), algumas vezes apresentando mais de uma cor ou contrastando com estruturas acessórias ou diásporos imaturos coloridos (Ridley 1930; Stiles 1982; Willson \& Thompson 1982). Os diásporos adaptados à dispersão por mamíferos, ao contrário, são odoríferos e de cores crípticas, como verde, amarelo, marrom e preto (van der Pijl 1982), e possuem casca normalmente mais espessa (Janson 1983).

O mutualismo estabelecido entre as plantas e seus dispersores, segundo alguns autores, se desenvolveu por coevolução difusa entre grupos de espécies variáveis ao longo do tempo e do espaço e não apenas da relação estreita entre duas espécies (Janzen 1980; Wheelwright \& Orians 1982; Howe 1984; Herrera 1995). As características morfológicas dos diásporos, em muitas famílias, mantiveram-se constantes durante a evolução das espécies vegetais, de modo que novos dispersores utilizam diásporos que originalmente foram selecionados por outros animais, havendo assim "exadaptação" (Howe 1984).

A morfologia das sementes está relacionada às diferentes estratégias de estabelecimento das plantas (Foster \& Janson 1985). Como padrão geral, sementes menores são produzidas em maiores quantidades (Harper et al. 1971) e permanecem dormentes no banco de sementes, necessitando de luz para se estabelecer. As sementes grandes, por possuírem mais reservas energéticas, são capazes de germinar em locais sombreados, formando um banco de plântulas, até o surgimento de situações propícias para o seu crescimento (Piña-Rodrigues et al. 1990; Leishman et al. 2000). Estas características ocorrem em espécies de estádios sucessionais iniciais e tardios, respectivamente, e recebem diversas denominações, tais como pioneiras e climácicas (Budowski 1965; 1970), colonizadoras e competidoras (Muller-Landau et al. 2002). A delimitação destes grupos, entretanto, nem sempre é clara, havendo muitas espécies de características intermediárias (Foster \& Janson 1985; Bullock 2000).

O padrão morfológico dos diásporos de uma determinada comunidade, portanto, pode elucidar diversos aspectos ecológicos de interação com a fauna local. Esta, mesmo não tendo necessariamente evoluído com a flora local, é determinante para a sua chegada, estabelecimento e permanência na comunidade.

Neste trabalho são descritos e sumarizados aspectos morfológicos de diásporos de árvores e arvoretas zoocóricas em uma região conservada, no sul do Brasil, com o objetivo de relacionar características relevantes para a dispersão, como tamanho, formato e cor dos diásporos, com aspectos relacionados ao estabelecimento de plântulas, como massa e quantidade de sementes. A hipótese levantada foi de que a morfologia dos diásporos apresenta padrões relacionados com as estratégias de dispersão e estabelecimento das espécies. Foram formuladas as seguintes questões: as espécies podem ser agrupadas de acordo com as características dos seus diásporos? Quais adaptações são mais abundantes e a que grupo de vertebrados podem estar relacionadas? Quais as possíveis implicações para o estabelecimento das sementes?

\section{Material e métodos}

Local de estudo - O Parque Estadual de Itapuã (PEI) ( $30^{\circ} 21^{\prime} \mathrm{S}$ - $51^{\circ} 03^{\prime} \mathrm{W}$; $30^{\circ} 26^{\prime} \mathrm{S}$ - 50 $\left.54^{\prime} \mathrm{W}\right)$, Viamão, Rio Grande do Sul, Brasil, compreende 5.566 ha, que margeiam o lago Guaíba e a laguna dos Patos, com altitudes que variam de cinco a $263 \mathrm{~m}$. As formas do relevo estão associadas a duas províncias geomorfológicas: o Escudo Cristalino Sul-RioGrandense, formado no Pré-Cambriano, e a Planície Costeira, oriunda do Neogeno (Quaternário) recente. Os solos, derivados da intemperização do granito ou de sedimentos arenosos, são bastante variados quanto à fertilidade e drenagem. O clima regional é subtropical úmido (Cfa de Köppen). A temperatura média anual é $17,5^{\circ} \mathrm{C}$ e a temperatura média do mês mais quente $22^{\circ} \mathrm{C}$. A precipitação anual varia de 1.100 a $1.300 \mathrm{~mm}$, com chuvas bem distribuídas durante o ano e sem estação seca. A área do Parque compreende grande 
diversidade de tipos fisionômicos e florísticos, como florestas (estacional nas encostas e de restinga na planície), campos rupestres, vegetação palustre e de dunas (Rio Grande do Sul 1997). Segundo Veloso e Góes Filho (1982), a região está inserida em uma zona de transição entre savana e floresta estacional, mantendo elementos característicos de ambas.

O estudo foi realizado principalmente em floresta estacional de encosta, no morro do Campista (altitude máxima de $174 \mathrm{~m}$ ), e floresta de restinga, nas praias de Fora e do Tigre. Estas áreas apresentam mínima intervenção humana e têm acesso exclusivo para pesquisas científicas (Rio Grande do Sul 1997). Eventualmente, foram realizadas coletas adicionais nas proximidades da lagoa Negra e nas praias da Pedreira e do Araçá.

A fauna de vertebrados é relativamente diversificada, sendo identificadas no plano de manejo do PEI 23 espécies de mamíferos e 37 espécies de répteis (Rio Grande do Sul 1997), embora este número possivelmente esteja subestimado, além de 219 espécies de aves (Scherer et al. 2007). Os potenciais dispersores de sementes incluem o bugio-ruivo (Alouatta guariba, Humboldt 1812), mão-pelada (Procyon cancrivorous, F.Cuvier 1798), graxains (Pseudalopex gymnocercus, Fischer 1914, e Cerdocyon thous, Linnaeus 1766), várias espécies de morcegos, lagarto-de-papo-amarelo (Tupinambis teguixin, Linnaeus 1758) (Rio Grande do Sul 1997). As aves que incluem frutos na dieta são em sua maioria de pequeno porte e de hábitos alimentares generalistas como sabiás (Turdus amaurochalinus, Cabains 1850; T. rufiventris, Vieillot 1818; T. albicolis, Vieillot 1818; Mimus saturninus, Lichtenstein 1823), bem-te-ví (Pitangus sulfuratus, Lineu 1766), sanhaço (Thraupis sayaca, Linnaeus 1976), guaracavas (Elaenia spp.), e poucas aves maiores como aracuã (Ortalis guttata, Spix 1825) e jacuaçu (Penelope obscura, Temminck 1815), além de espécies granívoras que eventualmente dispersam sementes como tico-ticos (Coryphospingus cucullatus, Muller 1776; Zonotrichia capensis, Müller 1776) e trinca-ferro (Saltator similis, d'Orbigny \& Lafresnaye 1837) (Scherer et al. 2007).

Coleta de dados - Foram incluídas no estudo as espécies de árvores e arvoretas com diásporos endozoocóricos, considerados como aqueles que apresentam estruturas carnosas que servem de recurso para animais frugívoros, encontrados em frutificação durante o período de estudo. Os diásporos foram coletados de junho/2003 a abril/2005, em caminhadas quinzenais ao longo de trilhas no morro do Campista, nas praias de Fora e do Tigre, e em visitas menos frequientes a outros pontos do PEI. Diásporos maduros foram coletados diretamente da copa, acondicionados em potes plásticos com identificação da árvore-matriz e mantidos resfriados em bolsa térmica e posteriormente em geladeira, até a análise em laboratório, em geral no dia seguinte à coleta. Os critérios para a detecção dos diásporos maduros foram variáveis, como fácil desprendimento do diásporo, deiscência do fruto e amolecimento da polpa, que puderam ser determinados pela visitação regular até o término da frutificação.

Em laboratório, foram retirados de cinco a 30 diásporos por pote, ao acaso, provenientes de uma a seis árvores matrizes, de acordo com a produtividade e abundância de indivíduos na área, e registrados a massa fresca, o tamanho (comprimento e maior diâmetro) e o número e massa de sementes por diásporo. Como comprimento, foi considerada a medida longitudinal ao eixo de ligação do diásporo à planta. As medidas de massa e comprimento foram tomadas respectivamente com o auxílio de balança de precisão $(0,0001 \mathrm{~g})$ e paquímetro $(0,01 \mathrm{~cm})$.

As árvores e arbustos que tiveram os frutos coletados foram identificados com uso de literatura taxonômica ou por comparação com exsicatas determinadas no Herbário ICN. As espécies foram agrupadas nas famílias reconhecidas pelo APG II (2003). Uma coleção das espécies estudadas foi incorporada ao acervo do Herbário ICN, como material testemunho.

Análise dos dados - A coloração dos diásporos maduros foi classificada, com alterações, de acordo com as categorias propostas por Willson et al. (1989): preto, vermelho, laranja, amarelo, branco, verde/ marrom, azul e bicolorido morfológico. Na categoria "preto" foram incluídos também os diásporos de coloração atropurpúrea, na "branco" os diásporos de colorações claras, e na categoria "bicolorido morfológico" as espécies com diásporos contrastantes com estruturas adjacentes conspícuas, como receptáculos florais coloridos. A ocorrência de coloração polimórfica entre indivíduos foi acrescentada à classificação e a cor azul não foi registrada. A massa e o comprimento dos diásporos e o número e massa de sementes por diásporo, em valores médios, foram ordenados através do método de médias recíprocas ("reciprocal averaging") (Hill 1973). A vantagem do método resulta da ordenação simultânea de unidades 
amostrais e variáveis, permitindo avaliar visualmente a relação entre ambas. As espécies (unidades amostrais) foram agrupadas no diagrama de ordenação de acordo com a semelhança entre as características descritivas, representadas pelas variáveis morfométricas (ter Braak 1995). Foi utilizado como valor para separação de grupos o diâmetro $1,2 \mathrm{~cm}$, uma vez que diásporos menores são associados à dispersão por aves pequenas e grandes, enquanto os maiores são associados a aves grandes ou a outros vertebrados (Wheelwright 1985).

Foram analisadas as relações entre o diâmetro e comprimento de diásporos e entre a razão massa das sementes/massa do diásporo $\left(\mathrm{R}_{\mathrm{SS} / \mathrm{D}}\right)$, com o comprimento. Os diásporos foram relacionados à síndrome de ornitocoria quando o comprimento foi inferior a $2 \mathrm{~cm}$, e à mamaliocoria quando apresentaram comprimento igual ou maior que $2 \mathrm{~cm}$ (Willson et al. 1989).

\section{Resultados}

Foram coletados diásporos de 64 espécies de árvores e arvoretas pertencentes a 48 gêneros e 29 famílias, que apresentaram adaptações a endozoocoria (Tab. 1). O comprimento médio dos diásporos para todas as espécies foi de $1,70 \pm 1,67 \mathrm{~cm}(\mathrm{n}=1.402)$, com a medida mínima de $0,3 \mathrm{~cm}$ para Casearia silvestris e a máxima de $8,5 \mathrm{~cm}$ para Inga vera. A massa média dos diásporos foi de 4,28 $\pm 12,0 \mathrm{~g}$ ( $\mathrm{n}=1.402)$, sendo o mínimo 0,023 g para Zanthoxylum rhoifolium e o máximo de 92,1 g para Cereus hildmannianus. O número de sementes variou de uma (32 espécies) a mais de 1.000 , para $C$. hildmannianus e Cecropia pachystachya. A razão $R_{\mathrm{SS} / \mathrm{D}}$ foi $0,35 \pm$ 0,21, com o mínimo de 0,03 para $C$. hildmannianus e o máximo de 0,96 para $Z$. hyemale.

Os diásporos de coloração cinza-azulada de Styrax leprosum e Symplocos spp., branco-rosado de Casearia decandra e verde-leitoso de Lithraea brasiliensis foram incluídos na categoria branco. Três espécies de Myrtaceae foram classificadas como pertencentes à categoria polimórfico (Blepharocalyx salicifolius, Myrcia palustris e Myrciaria cuspidata), por apresentarem diásporos maduros nas colorações laranja, vermelha ou roxa, em diferentes indivíduos. As categorias de cor mais freqüentes foram preto $(31,3 \%)$ e bicolorido (17,2\%; Fig. 1). Diásporos menores que 2,0 cm compr. apresentaram todas as colorações, enquanto os maiores foram principalmente amarelos ou verde/marrons, além de laranjas, pretos ou bicoloridos.
Os autovalores gerados pela ordenação através de médias recíprocas (eixo $1=0,445$ e eixo $2=0,073$ ) foram baixos, denotando gradientes curtos (ter Braak 1995), ou seja, uma variação gradativa das características analisadas para as diferentes espécies, sem formação de grupos isolados. De acordo com o número e massa de sementes e as dimensões dos diásporos delimitaram-se quatro grupos no diagrama de ordenação (Fig. 2). Os dois primeiros grupos (G1 e G2) foram contínuos e apresentaram de uma a três sementes por diásporo, sendo que o G1 incluiu espécies com diásporos de diâmetro médio menor que $1,2 \mathrm{~cm}$, e o G2 as com diásporos de tamanhos superiores. $\mathrm{O}$ terceiro e quarto grupos (G3 e G4) apresentaram mais do que três sementes, sendo que o G3 incluiu espécies com sementes pesadas ( $>0,01 \mathrm{~g})$ e o $\mathrm{G} 4$ espécies com sementes leves $(<0,01 \mathrm{~g})$. O G1 apresentou a maior riqueza (35 spp.), seguido pelo G2 (15), G3 (sete) e G4 (sete). Quanto à coloração, o G1 foi caracterizado por um grande número de espécies com diásporos pretos e vermelhos e ausência de diásporos verdes e amarelos. Foram exclusivos deste grupo diásporos brancos ou polimórficos, enquanto bicoloridos ocorreram na transição para o G2. No G3, ocorreu baixa variedade de colorações, com diásporos amarelos, pretos ou verdes. No G2 e G4 houve maior variedade de colorações. O diagrama de ordenação mostrou gradual diminuição da freqüência de diásporos pretos e vermelhos e um aumento de amarelos e verdes concomitantes ao aumento de massa, enquanto os diásporos de coloração laranja possuem distribuição sem tendência aparente para um dos grupos.

A maior parte das espécies (52) apresentou diásporos menores que $2,0 \mathrm{~cm}$ de comprimento. Dentre estas, $75 \%$ (39) apresentaram diâmetro inferior a $1,2 \mathrm{~cm}$ e $86,5 \%$ (45) poucas sementes (G1 e G2). Dentre as espécies com diásporos grandes, $58 \%$ (oito) apresentaram mais de três sementes (G3 e G4). Houve predominância de diásporos alongados no $\mathrm{G} 1$, com $71 \%$ das espécies (25), no G2, com 53\% (oito), e no G3, com $57 \%$ (quatro); e de diásporos achatados no G4, com 57\% (quatro) (Fig. 3A). Quanto à proporção da massa do diásporo alocado em sementes $\left(\mathrm{R}_{\mathrm{SS} / \mathrm{D}}\right)$, a maior parte das espécies se concentrou na faixa de 25 a $50 \%$, tanto para aquelas com diásporos pequenos (22), quanto com grandes (oito); 22 espécies ficaram na faixa de até $25 \%$ e massas superiores a $50 \%$ em sementes foram exclusivas de diásporos pequenos (10). As espécies com muitas sementes (G3 e G4) apresentaram a massa das sementes contribuindo com menos de 50\% da massa do diásporo (Fig. 3B). 
Tabela 1. Árvores e arvoretas dispersadas por vertebrados no Parque Estadual de Itapuã, Viamão, RS, Brasil, com respectivas características morfológicas, em valores médios e desvio padrão, e coloração dos diásporos. N, número de diásporos analisados; $\mathrm{MD}$, massa do diásporo; $\mathrm{CD}$, comprimento e, $\mathrm{DD}$, diâmetro do diásporo; NS, número de sementes médio, máximo e mínimo; MS, massa individual das sementes; MSS, massa do conjunto de sementes por diásporo. Coloração: A, amarelo; B, branco; Bc, bicolorido; L, laranja; P, preto; Pm, polimórfico; V, vermelho; V/M, verde/marrom.

\begin{tabular}{|c|c|c|c|c|c|c|c|c|}
\hline Famílias/Espécies & Coloração & $\mathrm{N}$ & $\mathrm{MD}(\mathrm{g})$ & $\mathrm{CD}(\mathrm{cm})$ & $\mathrm{DD}(\mathrm{cm})$ & NS & $\operatorname{MS}(\mathrm{g})$ & $\operatorname{MSS}(\mathrm{g})$ \\
\hline \multicolumn{9}{|l|}{ ANACARDIACEAE } \\
\hline Lithraea brasiliensis Marchand & B & 20 & $0,064 \pm 0,006$ & $0,57 \pm 0,01$ & $0,62 \pm 0,01$ & 1 & $0,033 \pm 0,003$ & $0,033 \pm 0,003$ \\
\hline Schinus molle $\mathrm{L}$ & $\mathrm{V}$ & 20 & $0,04 \pm 0,004$ & $0,52 \pm 0,02$ & $0,5 \pm 0,02$ & 1 & $0,016 \pm 0,002$ & $0,016 \pm 0,002$ \\
\hline S. polygamus (Cav.) Cabrera & $\mathrm{P}$ & 12 & $0,048 \pm 0,006$ & $0,46 \pm 0,02$ & $0,5 \pm 0,03$ & 1 & $0,015 \pm 0,005$ & $0,015 \pm 0,005$ \\
\hline \multicolumn{9}{|l|}{ ANNONACEAE } \\
\hline Rollinia maritima Záchia & A & 23 & $2,8 \pm 0,8$ & $1,51 \pm 0,20$ & $2,03 \pm 0,22$ & $11,5(5-18)$ & $0,062 \pm 0,022$ & $0,68 \pm 0,25$ \\
\hline R. silvatica (A. St.-Hil.) Mart. & A & 20 & $19,7 \pm 8,0$ & $3,17 \pm 0,51$ & $3,57 \pm 0,52$ & $26,5(8-38)$ & $0,21 \pm 0,03$ & $5,2 \pm 2,4$ \\
\hline \multicolumn{9}{|l|}{ AQUIFOLIACEAE } \\
\hline Ilex dumosa Reissek & $\mathrm{P}$ & 26 & $0,071 \pm 0,010$ & $0,41 \pm 0,04$ & $0,52 \pm 0,04$ & $4,1(4-5)$ & $0,0043 \pm 0,0014$ & $0,017 \pm 0,003$ \\
\hline \multicolumn{9}{|l|}{ ARECACEAE } \\
\hline Butia capitata (Mart.) Becc. & $\mathrm{L}$ & 30 & $7,5 \pm 3,3$ & $1,87 \pm 0,22$ & $2,53 \pm 0,37$ & 1 & $1,4 \pm 0,5$ & $1,4 \pm 0,5$ \\
\hline Syagrus romanzoffiana (Cham.) Glassman & $\mathrm{L}$ & 15 & $3,4 \pm 0,3$ & $1,97 \pm 0,07$ & $1,65 \pm 0,05$ & 1 & $1,5 \pm 0,1$ & $1,5 \pm 0,1$ \\
\hline \multicolumn{9}{|l|}{ BORAGINACEAE } \\
\hline Cordia ecalyculata Vell. & $\mathrm{V}$ & 25 & $2,1 \pm 0,5$ & $1,55 \pm 0,09$ & $1,5 \pm 0,15$ & 1 & $0,34 \pm 0,13$ & $0,34 \pm 0,13$ \\
\hline \multicolumn{9}{|l|}{ CACTACEAE } \\
\hline Cereus hildmannianus K. Schum. & $\mathrm{Bc}(\mathrm{AB})$ & 10 & $92,1 \pm 42,7$ & $6,09 \pm 1,12$ & $5,57 \pm 1,01$ & $>1000$ & $0,0014 \pm 0,0003$ & $3,4 \pm 1,5$ \\
\hline \multicolumn{9}{|l|}{ CANNABACEAE } \\
\hline Trema micrantha (L.) Blume & $\mathrm{V}$ & 30 & $0,026 \pm 0,003$ & $0,41 \pm 0,03$ & $0,34 \pm 0,03$ & 1 & $0,0047 \pm 0,0009$ & $0,0047 \pm 0,0009$ \\
\hline \multicolumn{9}{|l|}{ CLUSIACEAE } \\
\hline Garcinia gardneriana (Planch. \& Triana) Zappi & A & 25 & $14,3 \pm 6,6$ & $3,64 \pm 0,47$ & $2,72 \pm 0,51$ & $1,9(1-3)$ & $2,6 \pm 0,7$ & $4,8 \pm 2,2$ \\
\hline \multicolumn{9}{|l|}{ EBENACEAE } \\
\hline Diospyros inconstans Jacq. & $\mathrm{P}$ & 30 & $4,3 \pm 1,4$ & $1,71 \pm 0,14$ & $1,99 \pm 0,26$ & $3,4(1-6)$ & $0,49 \pm 0,12$ & $1,5 \pm 0,7$ \\
\hline \multicolumn{9}{|l|}{ ERYTHROXYLACEAE } \\
\hline Erythroxylum argentinum O.E. Schulz & $\mathrm{V}$ & 22 & $0,2 \pm 0,03$ & $0,97 \pm 0,05$ & $0,59 \pm 0,04$ & 1 & $0,082 \pm 0,059$ & $0,082 \pm 0,059$ \\
\hline \multicolumn{9}{|l|}{ EUPHORBIACEAE } \\
\hline Sapium glandulosum (L.) Morong & $\mathrm{Bc}(\mathrm{VP})$ & 18 & $0,1 \pm 0,01$ & $0,68 \pm 0,04$ & $0,61 \pm 0,04$ & 1 & $0,063 \pm 0,006$ & $0,063 \pm 0,006$ \\
\hline \multicolumn{9}{|l|}{ FABACEAE } \\
\hline Enterolobium contortisiliquum (Vell.) Morong & $\mathrm{P}$ & 12 & $15 \pm 4,2$ & $8,53 \pm 0,64$ & $4,43 \pm 0,72$ & $7,7(5-10)$ & $0,19 \pm 0,03$ & $5,6 \pm 6,3$ \\
\hline Inga vera Willd. & $\mathrm{V} / \mathrm{M}$ & 5 & $11,8 \pm 4,5$ & $5,57 \pm 0,97$ & $2,05 \pm 0,18$ & $14,6(10-20)$ & $1 \pm 0,2$ & $7,2 \pm 2,5$ \\
\hline \multicolumn{9}{|l|}{ LAMIACEAE } \\
\hline Vitex megapotamica (Spreng.) Moldenke & $\mathrm{P}$ & 20 & $2,38 \pm 0,29$ & $1,63 \pm 0,09$ & $1,63 \pm 0,07$ & 1 & $0,33 \pm 0,03$ & $0,33 \pm 0,03$ \\
\hline \multicolumn{9}{|l|}{ LAURACEAE } \\
\hline Aiouea saligna Meisn. & $\mathrm{Bi}(\mathrm{PV})$ & 20 & $0,98 \pm 0,14$ & $1,24 \pm 0,08$ & $1,13 \pm 0,07$ & 1 & $0,32 \pm 0,07$ & $0,32 \pm 0,07$ \\
\hline Ocotea indecora (Schott) Mez & $\mathrm{Bi}(\mathrm{PV})$ & 17 & $0,48 \pm 0,16$ & $1,13 \pm 0,14$ & $0,81 \pm 0,06$ & 1 & $0,19 \pm 0,1$ & $0,19 \pm 0,10$ \\
\hline O. puberula (Rich.) Nees & $\mathrm{Bi}(\mathrm{PV})$ & 15 & $1,2 \pm 0,17$ & $1,44 \pm 0,08$ & $1,31 \pm 0,28$ & 1 & $0,45 \pm 0,11$ & $0,45 \pm 0,11$ \\
\hline O. pulchella (Nees.) Mez & $\mathrm{P}$ & 30 & $0,25 \pm 0,03$ & $1,13 \pm 0,07$ & $0,62 \pm 0,03$ & 1 & $0,14 \pm 0,02$ & $0,14 \pm 0,02$ \\
\hline
\end{tabular}




\begin{tabular}{|c|c|c|c|c|c|c|c|c|}
\hline Famílias/Espécies & Coloração & $\mathrm{N}$ & $\mathrm{MD}(\mathrm{g})$ & $\mathrm{CD}(\mathrm{cm})$ & $\mathrm{DD}(\mathrm{cm})$ & NS & MS (g) & MSS (g) \\
\hline \multicolumn{9}{|l|}{ MELIACEAE } \\
\hline Cabralea canjerana (Vell.) Mart. & $\mathrm{Bi}(\mathrm{VB})$ & 13 & $0,97 \pm 0,26$ & $1,91 \pm 0,32$ & $1,05 \pm 0,05$ & $1,5(1-2)$ & $0,47 \pm 0,09$ & $0,7 \pm 0,22$ \\
\hline Trichilia claussenii C. DC. & $\mathrm{Bi}(\mathrm{VP})$ & 16 & $1,1 \pm 0,11$ & $1,5 \pm 0,12$ & $1,12 \pm 0,05$ & 1 & $0,81 \pm 0,27$ & $0,81 \pm 0,10$ \\
\hline T. elegans A. Juss. & $\mathrm{Bi}(\mathrm{VP})$ & 20 & $0,062 \pm 0,039$ & $1,4 \pm 0,15$ & $0,66 \pm 0,10$ & $1,5(1-2)$ & $0,037 \pm 0,18$ & $0,044 \pm 0,031$ \\
\hline \multicolumn{9}{|l|}{ MORACEAE } \\
\hline Ficus cestrifolia Schott & $\operatorname{Pm}(\mathrm{PVB})$ & 20 & $0,82 \pm 0,12$ & $1,13 \pm 0,03$ & $1,13 \pm 0,07$ & $80,2(35-110)$ & $0,0009 \pm 0,0002$ & $0,072 \pm 0,039$ \\
\hline Sorocea bonplandii (Baill.) W.C. Burger et al. & $\mathrm{Bi}(\mathrm{PV})$ & 30 & $1,8 \pm 0,4$ & $1,56 \pm 0,1$ & $1,42 \pm 0,08$ & 1 & $0,29 \pm 0,16$ & $0,29 \pm 0,16$ \\
\hline \multicolumn{9}{|l|}{ MYRSINACEAE } \\
\hline Myrsine coriacea (Sw.) R. Br. & $\mathrm{P}$ & 25 & $0,043 \pm 0,008$ & $0,41 \pm 0,02$ & $0,44 \pm 0,02$ & 1 & $0,021 \pm 0,005$ & $0,021 \pm 0,005$ \\
\hline M. guianensis (Aubl.) Kuntze & $\mathrm{P}$ & 20 & $0,15 \pm 0,01$ & $0,59 \pm 0,02$ & $0,63 \pm 0,03$ & 1 & $0,073 \pm 0,006$ & $0,073 \pm 0,006$ \\
\hline \multicolumn{9}{|l|}{ MYRTACEAE } \\
\hline Blepharocalyx salicifolius (Kunth) O. Berg & Pm (PVL) & 30 & $0,22 \pm 0,07$ & $0,7 \pm 0,09$ & $0,7 \pm 0,08$ & $1,5(1-3)$ & $0,036 \pm 0,012$ & $0,048 \pm 0,021$ \\
\hline Eugenia hiemalis Cambess. & $\mathrm{P}$ & 24 & $0,38 \pm 0,16$ & $1,06 \pm 0,16$ & $0,74 \pm 0,11$ & $1,1(1-2)$ & $0,14 \pm 0,04$ & $0,16 \pm 0,08$ \\
\hline E. myrcianthes Nied. & A & 27 & $11,9 \pm 5,7$ & $2,93 \pm 0,6$ & $2,98 \pm 0,49$ & $1,2(1-2)$ & $1,3 \pm 0,6$ & $1,6 \pm 0,9$ \\
\hline E. rostrifolia D. Legrand & $\mathrm{L}$ & 18 & $2,4 \pm 0,75$ & $1,78 \pm 0,19$ & $1,69 \pm 0,18$ & $1,3(1-2)$ & $0,5 \pm 0,8$ & $0,65 \pm 0,23$ \\
\hline E. speciosa Cambess. & $\mathrm{L}$ & 23 & $7 \pm 2,8$ & $2,51 \pm 0,51$ & $2,57 \pm 0,35$ & $1,1(1-2)$ & $2 \pm 1$ & $2,1 \pm 1,0$ \\
\hline E. uniflora $\mathrm{L}$. & $\mathrm{P}$ & 30 & $1,5 \pm 0,3$ & $1,16 \pm 0,06$ & $1,64 \pm 0,12$ & $1,1(1-2)$ & $0,2 \pm 0,07$ & $0,22 \pm 0,06$ \\
\hline E. uruguayensis Cambess. & $\mathrm{P}$ & 10 & $0,96 \pm 0,29$ & $1,59 \pm 0,28$ & $1,02 \pm 0,11$ & $1,2(1-2)$ & $0,19 \pm 0,04$ & $0,22 \pm 0,08$ \\
\hline Myrcia palustris DC. & Pm (PV) & 30 & $0,43 \pm 0,12$ & $0,82 \pm 0,08$ & $0,9 \pm 0,1$ & $2,5(1-4)$ & $0,044 \pm 0,011$ & $0,1 \pm 0,03$ \\
\hline M. selloi (Spreng.) N. Silveira & $\mathrm{P}$ & 30 & $0,2 \pm 0,11$ & $0,58 \pm 0,13$ & $0,7 \pm 0,15$ & $1,2(1-2)$ & $0,029 \pm 0,018$ & $0,037 \pm 0,025$ \\
\hline Myrcianthes pungens (O. Berg.) D. Legrand & $\mathrm{P}$ & 23 & $3,2 \pm 0,9$ & $1,64 \pm 0,18$ & $1,79 \pm 0,19$ & $1,7(1-3)$ & $0,23 \pm 0,05$ & $0,41 \pm 0,17$ \\
\hline Myrciaria cuspidata $\mathrm{O}$. Berg & Pm (PVL) & 30 & $0,5 \pm 0,07$ & $0,9 \pm 0,05$ & $0,94 \pm 0,06$ & 1 & $0,098 \pm 0,017$ & $0,098 \pm 0,017$ \\
\hline Myrrhinium atropurpureum Schott & $\mathrm{P}$ & 25 & $0,17 \pm 0,03$ & $0,7 \pm 0,05$ & $0,61 \pm 0,05$ & $2,6(2-4)$ & $0,016 \pm 0,003$ & $0,045 \pm 0,010$ \\
\hline Psidium cattleianum Sabine & A & 20 & $12,2 \pm 5,4$ & $3,02 \pm 0,57$ & $2,82 \pm 0,38$ & $43,6(3-82)$ & $0,016 \pm 0,008$ & $0,63 \pm 0,25$ \\
\hline \multicolumn{9}{|l|}{ NYCTAGINACEAE } \\
\hline Guapira opposita (Vell.) Reitz & $\mathrm{Bc}(\mathrm{PV})$ & 25 & $0,61 \pm 0,15$ & $1,18 \pm 0,20$ & $0,88 \pm 0,04$ & 1 & $0,17 \pm 0,03$ & $0,17 \pm 0,03$ \\
\hline \multicolumn{9}{|l|}{ ROSACEAE } \\
\hline Prunus myrtifolia (L.) Urb. & $\mathrm{L}$ & 30 & $0,85 \pm 0,08$ & $1,09 \pm 0,02$ & $1,2 \pm 0,04$ & 1 & $0,53 \pm 0,05$ & $0,53 \pm 0,05$ \\
\hline \multicolumn{9}{|l|}{ RUBIACEAE } \\
\hline Chomelia obtusa Cham. \& Schltdl. & $\mathrm{P}$ & 20 & $0,18 \pm 0,04$ & $0,9 \pm 0,05$ & $0,66 \pm 0,07$ & 1 & $0,028 \pm 0,008$ & $0,028 \pm 0,008$ \\
\hline Guettarda uruguensis Cham. \& Schltdl. & $\mathrm{P}$ & 20 & $0,57 \pm 0,15$ & $1,12 \pm 0,15$ & $1,06 \pm 0,12$ & 1 & $0,17 \pm 0,03$ & $0,17 \pm 0,03$ \\
\hline Randia ferox (Cham. \& Schltdl.) DC. & A & 5 & $12,5 \pm 5,3$ & $2,95 \pm 0,58$ & $2,86 \pm 0,5$ & $29,5(24-32)$ & $0,094 \pm 0,63$ & $4,4 \pm 3,7$ \\
\hline \multicolumn{9}{|l|}{ RUTACEAE } \\
\hline Zanthoxylum fagara (L.) Sarg. & $\mathrm{P}$ & 20 & $0,024 \pm 0,006$ & $0,35 \pm 0,04$ & $0,32 \pm 0,02$ & 1 & $0,024 \pm 0,006$ & $0,023 \pm 0,006$ \\
\hline Z. rhoifolium Lam. & $\mathrm{P}$ & 15 & $0,023 \pm 0,001$ & $0,35 \pm 0,02$ & $0,35 \pm 0,02$ & 1 & $0,022 \pm 0,002$ & $0,022 \pm 0,002$ \\
\hline \multicolumn{9}{|l|}{ SALICACEAE } \\
\hline Banara parviflora (A. Gray) Benth. & $\mathrm{V}$ & 20 & $0,22 \pm 0,10$ & $0,64 \pm 0,09$ & $0,7 \pm 0,12$ & $6,9(1-29)$ & $0,0008 \pm 0,0002$ & $0,0092 \pm 0,0089$ \\
\hline Casearia decandra Jacq. & $\mathrm{B}$ & 30 & $0,28 \pm 0,08$ & $0,76 \pm 0,08$ & $0,77 \pm 0,09$ & $1,2(1-3)$ & $0,06 \pm 0,016$ & $0,073 \pm 0,029$ \\
\hline C. silvestris $\mathrm{Sw}$ & $\mathrm{V}$ & 30 & $0,028 \pm 0,023$ & $0,33 \pm 0,09$ & $0,41 \pm 0,11$ & $3,2(1-11)$ & $0,0017 \pm 0,0005$ & $0,0072 \pm 0,0088$ \\
\hline \multicolumn{9}{|l|}{ SAPINDACEAE } \\
\hline Allophylus edulis (A. St.-Hil. et al.) Radlk. & $\mathrm{V}$ & 30 & $0,24 \pm 0,03$ & $0,8 \pm 0,04$ & $0,72 \pm 0,04$ & 1 & $0,075 \pm 0,011$ & $0,075 \pm 0,011$ \\
\hline
\end{tabular}




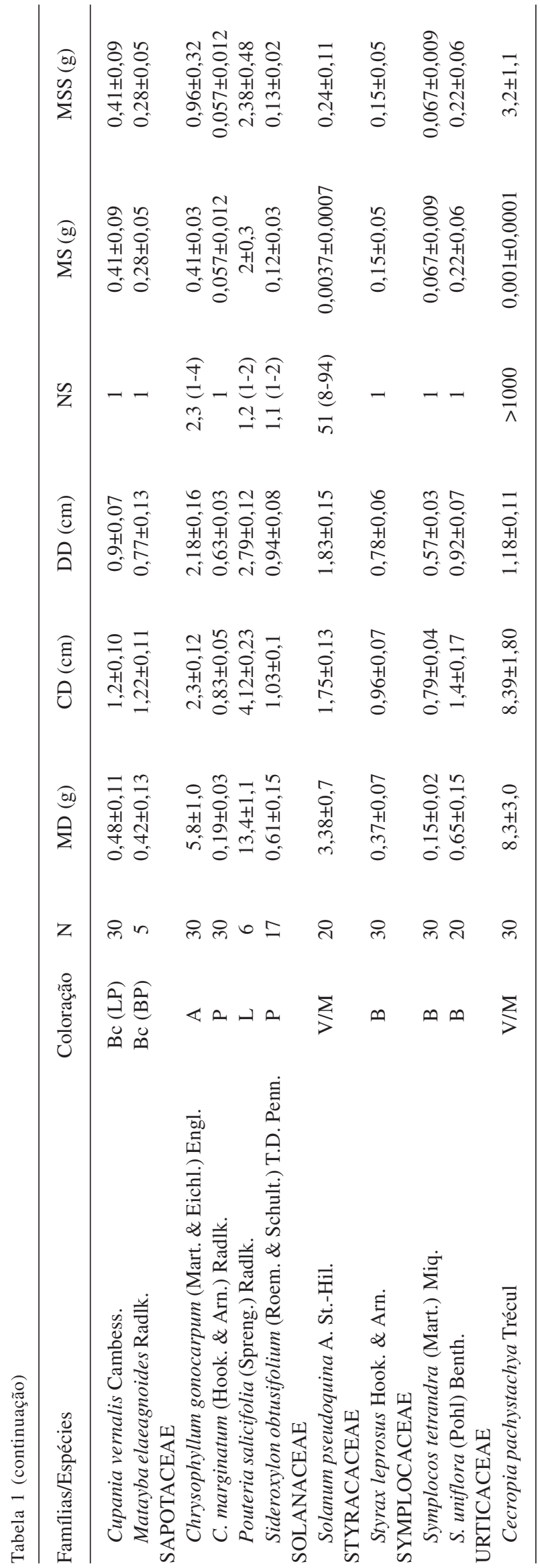

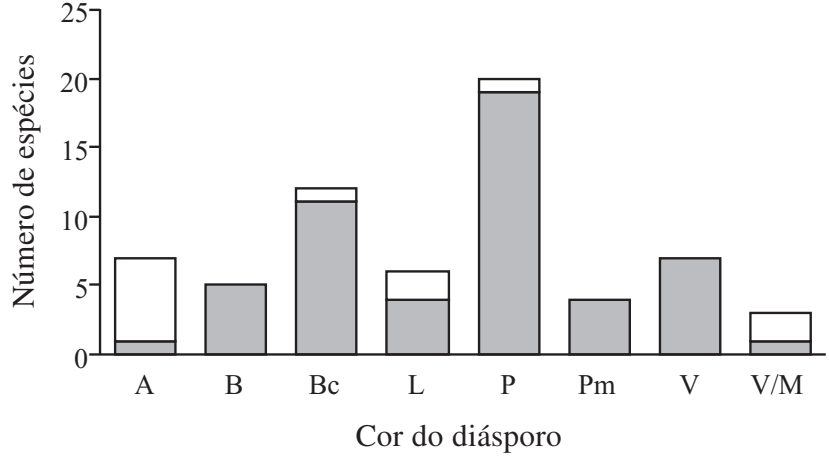

Figura 1. Cor dos diásporos de 64 espécies de árvores e arvoretas dispersos por vertebrados no Parque Estadual de Itapuã, RS, Brasil, com relação aos comprimentos médios dos diásporos ( $\square=<2,0 \mathrm{~cm}$ e $\square=>2,0 \mathrm{~cm}$ ). A, amarelo; B, branco; Bc, bicolorido; $\mathrm{L}$, laranja; $\mathrm{P}$, preto; Pm, polimórfico; V, vermelho; V/M, verde/ marrom.

\section{Discussão}

Os valores médios de comprimento e diâmetro dos diásporos foram baixos na vegetação estudada, o que se deve ao grande número de espécies ornitocóricas. Este predomínio é condizente com padrões encontrados na Mata Atlântica (F. Campassi, com. pessoal) e pronunciados em locais com temperaturas mais baixas, em maiores latitudes. Este padrão é também comum em outras regiões subtropicais como em Hong Kong (Corlett 1996) e em localidades na Austrália (Willson et al. 1989), em que as aves representam importante papel como dispersoras. Fogem a este padrão as regiões de cerrado (Galetti 2001) e de savana africana (Knighth \& Siegfried 1983), nas quais se verifica maior frequiência de frutos mamaliocóricos, associados à presença de mamíferos de grande porte.

A estratégia mais frequiente entre as árvores e arvoretas zoocóricas das diferentes fisionomias do PEI foi a produção de diásporos pequenos, com poucas sementes e cores correspondentes à síndrome de ornitocoria. Diásporos pequenos com poucas sementes (G1) apresentaram colorações como preto, bicolorido e vermelho, coerentes com a síndrome de ornitocoria. Diásporos alongados com sementes compreendendo grande porcentagem da massa dos diásporos permitem a ingestão por mais espécies de aves frugívoras em comparação a diásporos de mesmo volume, porém esféricos (Mazer e Wheelwright 1993). Diásporos alongados podem ser vantajosos em ambientes florestais, onde há grande abundância de aves.

Diásporos de maior tamanho e com poucas sementes (G2) foram associados à síndrome mista e mamaliocórica (Jordano 1995). Neste grupo, os 


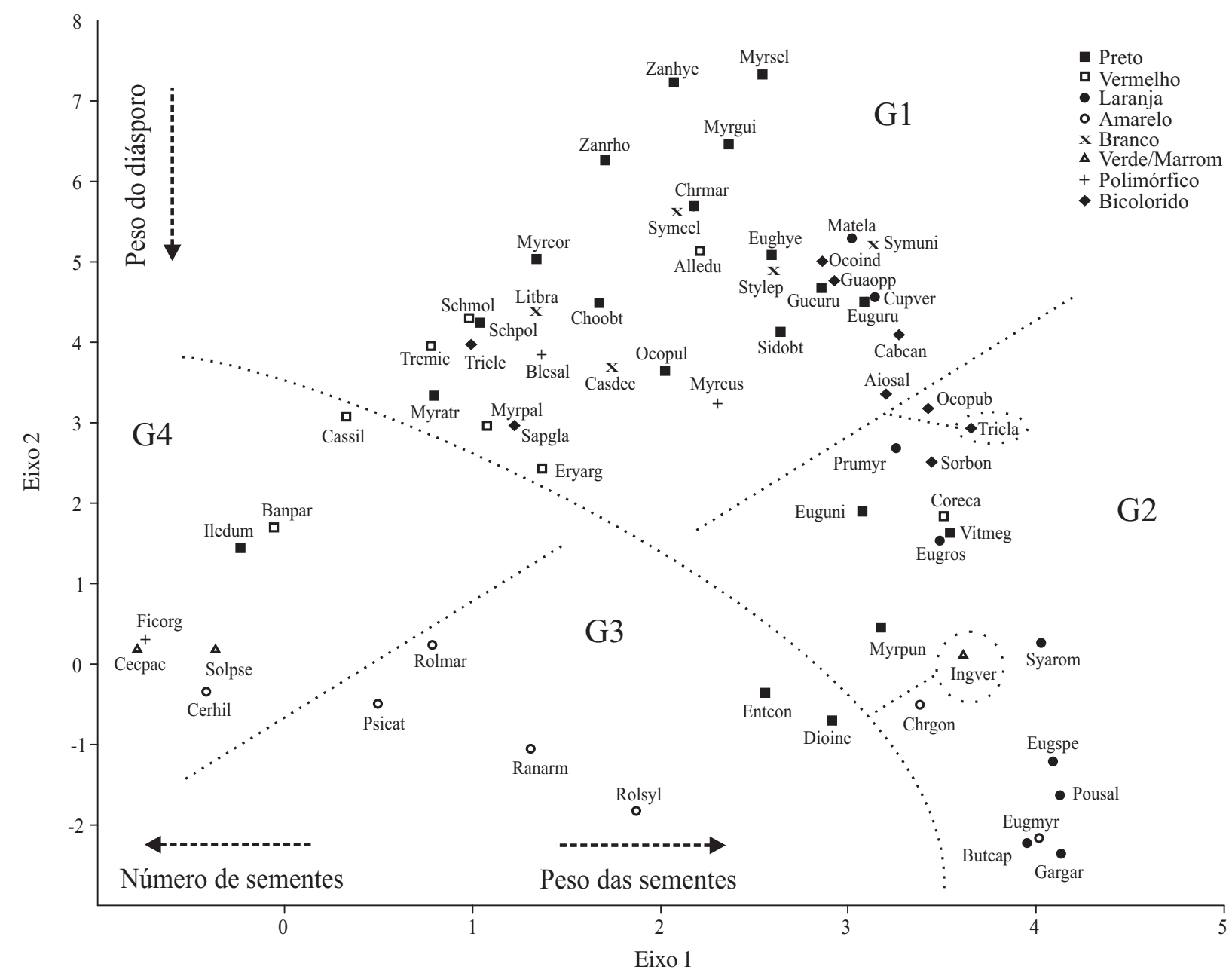

Figura 2. Ordenação de 64 espécies de árvores e arvoretas dispersadas por vertebrados no Parque Estadual de Itapuã, RS, Brasil, baseada na massa e comprimento dos diásporos e no número e massa das sementes por diásporo, em valores médios. As espécies foram classificadas segundo a coloração dos diásporos, com abreviaturas do gênero e epíteto específico de acordo com a Tab. 1. Características dos diásporos: $(\mathrm{G} 1)<1,2 \mathrm{~cm}$ de comprimento e 1-3 sementes; $(\mathrm{G} 2)>1,2 \mathrm{~cm}$ de comprimento e 1-3 sementes; $(\mathrm{G} 3)>3$ sementes pesadas $(>0,01 \mathrm{~g}) ;(\mathrm{G} 4)>3$ sementes leves $(>0,01 \mathrm{~g})$. Autovalores para o eixo $1=0,445$ e eixo $2=0,073$.

diásporos de menor massa apresentaram uma maior frequiência da coloração bicolorida, seguida da preta e da laranja, e ausência de exocarpo espesso, que são características relacionadas à dispersão por aves (Janson 1983). Entretanto, algumas destas espécies, como Sorocea bonplandii e Vitex megapotamica, são consumidas por mamíferos (G. Buss, com. pess.), que associado ao maior tamanho de diásporo, indica síndrome de dispersão mista. À medida que a massa dos diásporos aumenta, se tornam mais freqüentes as cores laranja e amarela e aparecem diásporos com casca espessa, como Pouteria salicifolia e Garcinia gardneriana, caracterizando a síndrome estritamente mamaliocórica (Janson 1983).

O grupo G3 reúne características quanto à coloração e à presença de casca espessa que enquadram as espécies na síndrome de mamaliocoria. Diásporos grandes vêm sendo associados à dispersão por vetores bióticos (Hughes et al. 1994; Tiffney 2004), enquanto aqueles com estruturas protetoras de modo geral restringem o consumo por aves (Janson 1983; Willson et al. 1989). Como exemplos no local, os diásporos de Randia ferox e Enterolobium contortisiliquum possuem casca espessa que os torna inacessíveis às aves. A separação dos grupos G2 e G3 baseia-se, principalmente, no fato deste último apresentar maior número de sementes por diásporo.

Uma ressalva importante para as 23 espécies com diásporos grandes e com alto investimento em sementes (G2 e G3) refere-se aos efeitos da degradação ambiental e da extinção local de vertebrados frugívoros. Segundo Corlett (1996) e Kitamura et al. (2002), 

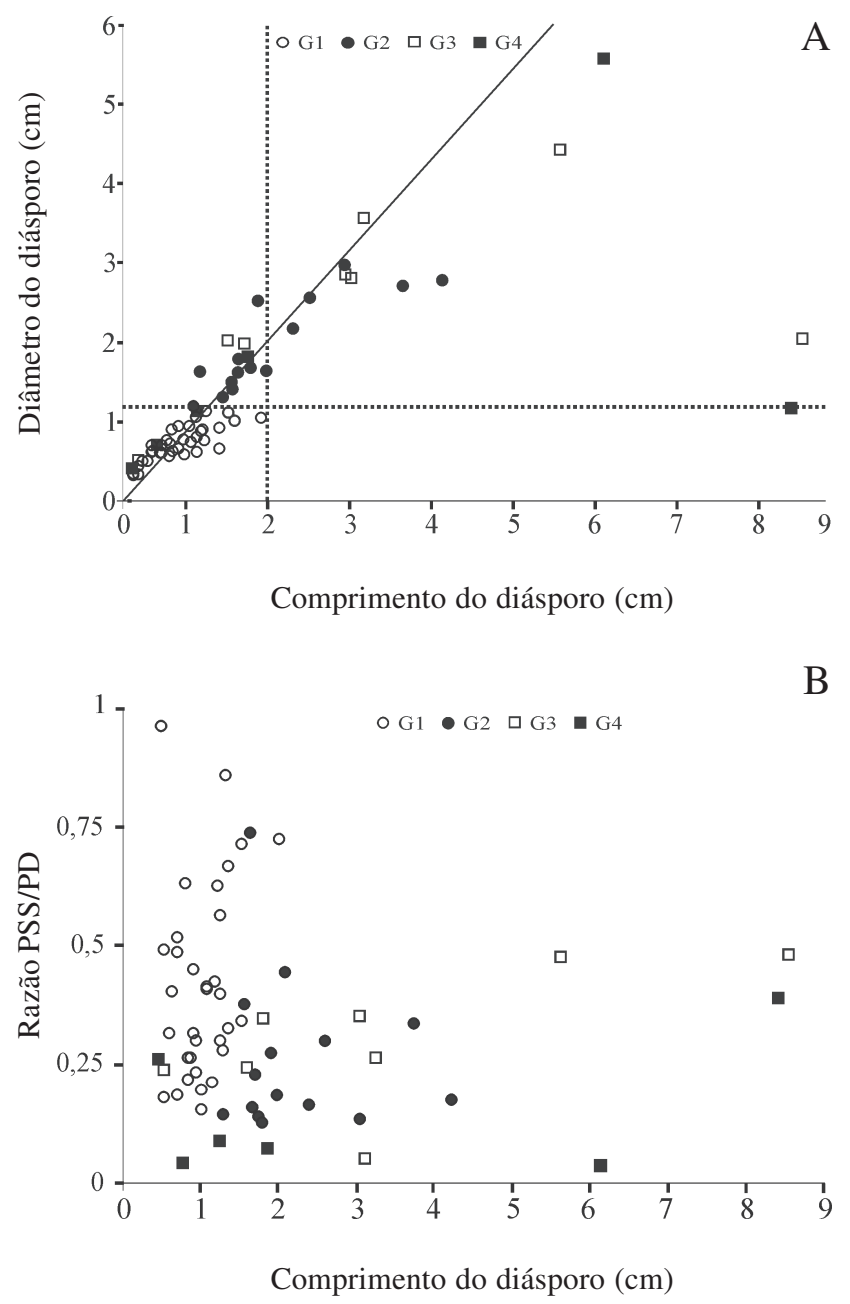

Figura 3. Comprimento dos diásporos de 64 espécies de árvores e arvoretas zoocóricas ocorrentes no Parque Estadual de Itapuã, RS, Brasil, com relação ao diâmetro (A) e a razão entre a massa das sementes e do diásporo $\left(\mathrm{R}_{\mathrm{SS} / \mathrm{D}}\right)(\mathrm{B})$. As espécies foram classificadas em grupos (G1, G2, G3 e G4) segundo o diagrama de ordenação (Fig. 2). Diásporos menores que $2 \mathrm{~cm}$ de comprimento são relacionados à síndrome de ornitocoria e os maiores a de mamaliocoria (Willson et al. 1989). (A) Diásporos situados acima da diagonal (21 spp.) apresentam formato achatado, na linha (4 spp.) formato esférico, e abaixo da diagonal (39 spp.) formato alongado. A linha horizontal no valor de 1,2 cm diâm. corresponde ao limite máximo de tamanho de diásporos dispersados por aves pequenas (Wheelwright 1985) e a linha vertical no valor de $2 \mathrm{~cm}$ compr. separa diásporos ornitocóricos e mamaliocóricos (Willson et al. 1989).

apenas os frugívoros de pequeno porte persistem em ambientes alterados, mas não são capazes de dispersar diásporos com sementes grandes. Assim sendo, as populações das espécies dos grupos G2 e G3 podem ser mais vulneráveis, conduzindo à necessidade de programas de recrutamento destas espécies. Discussões neste sentido vêm ocorrendo com relação aos riscos de extinção de espécies vegetais em fragmentos onde não existem mais dispersores de grande porte (Silva \& Tabarelli 2000; Galetti 2001).

A presença de muitas sementes pequenas $(\mathrm{G} 4)$ ocorreu tanto em espécies com coloração e tamanho de diásporo correspondente à ornitocoria (Casearia sylvestris, Banara parviflora, Ilex dumosa e Ficus cestrifolia), como à mamaliocoria (Cereus hildmannianus e Cecropia pachystachya, Solanum pseudoquina). A maior parte destas espécies, entretanto, pode ser dispersa por aves, devido à ausência de casca espessa ou pela deiscência dos frutos quando maduros (C. hildmannianus), que coincide, portanto, com a síndrome de dispersão mista. A baixa especificidade deste grupo provavelmente é uma característica favorável ao estabelecimento de espécies pioneiras, que devem investir na produção de muitas sementes para o incremento na probabilidade de ocupação de sítios favoráveis, representados principalmente por áreas em estádios iniciais de sucessão (Foster \& Janson 1985; Leishman et al. 2000).

As características morfológicas permitiram sumarizar diferenças nos diásporos das espécies de árvores e arvoretas zoocóricas do PEI, as quais foram atribuídas a pressões seletivas determinadas por diferentes estratégias de estabelecimento e pelo grupo de animais dispersores. Como principais conclusões tem-se que: (1) a característica 'poucas sementes' (G1 e G2) não parece ser relacionada a uma síndrome específica; (2) a separação destas espécies em diferentes síndromes é diretamente relacionada ao tamanho dos diásporos: a característica 'muitas sementes pesadas' (G3) é associada à síndrome de mamaliocoria, e 'muitas sementes pequenas' (G4) à síndrome mista; (3) os padrões encontrados se apresentaram contínuos, indicando uma exploração de nichos diversos quanto à dispersão e estabelecimento, embora a estratégia do G1 (diásporos pequenos e poucas sementes) tenha sido bastante uniforme e muito freqüente na área, provavelmente devido à relevância de pequenas aves como dispersoras de sementes no local de estudo e com maior proporção em massa de sementes por diásporo.

\section{Agradecimentos}

Ao $\mathrm{CNPq}$ pela Bolsa de Iniciação Científica (PIBIC/CNPq-UFRGS) à primeira autora e pelo apoio financeiro (CNPq processo 472960/2003-2); à SEMA/RS pela permissão e apoio logístico; ao Centro de Ecologia da UFRGS pelo empréstimo da balança de precisão; ao Departamento de Botânica pela infraestrutura disponibilizada. 


\section{Referências bibliográficas}

APG II (Angiosperm Phylogeny Group). 2003. An update of the Angiosperm Phylogeny Group classification for orders and families of flowering plants: APG II. Botanical Journal of the Linnean Society 141: 399-436.

Budowski, G. 1965. Distribution of tropical rain forest species in the light of successional process. Turialba 15: 40-42.

Budowski, G. 1970. The distinction between old secondary and climax species in tropical Central American lowland forests. Tropical Ecology 11: 44-48.

Bullock, J.M. 2000. Gaps and seedling colonization. Pp. 375-395. In: M. Fenner (ed.). Seeds: the ecology of regeneration in plant communities. London, CABI Publishing.

Corlett, R.T. 1996. Characteristics of vertebrate-dispersed fruits in Hong Kong. Journal of Tropical Ecology 12: 819-833.

Foster, S.A. \& Janson, C.H. 1985. The relationship between seed size and establishment conditions in tropical woody plants. Ecology 66: 773-780.

Galetti, M. 2001. The future of the Atlantic Forest. Conservation Biology 15: 4-5.

Geritz, S.A.H. 1998. Co-evolution of seed size and seed predation. Evolutionary Ecology 12: 891-911.

Hammond, D.S. \& Brown, V.K. 1995. Seed size of woody plants in relation to disturbance, soil type in wet neotropical forests. Ecology 76: 2544-2561.

Harper, J.L.; Lovell, P.H. \& Moore, K.G. 1971. The shapes and sizes of seeds. Annual Review of Ecology and Systematics 2: 327-356.

Herrera, C.M. 1995. Plant-vertebrate seed dispersal systems in the Mediterranean: ecological, evolutionary, and historical determinants. Annual Review of Ecology and Systematics 26: 705-727.

Hill, M.O. 1973. Reciprocal averaging: an eigenvector method of ordination. Journal of Ecology 61: 237-249.

Howe, H.F. 1984. Constraints on the evolution of mutualisms. The American Naturalist 123: 764-777.

Hughes, L.; Dunlop, M.; French, K.; Leishman, M.R.; Rice, B.; Rodgerson, L. \& Westoby, M. 1994. Predicting dispersal spectra: a minimal set of hypotheses based on plant attributes. Journal of Ecology 82: 933-950.

Janson, C.H. 1983. Adaptation of fruit morphology to dispersal agents in a Neotropical Forest. Science 219: 187-189.

Janson, C.H. 1992. Measuring evolutionary constraints: a Markov Model for phylogenetic transitions among seed dispersal syndromes. Evolution 46: 136-158.

Janzen, D.H. 1969. Seed-eaters versus seed size, number, toxicity, and dispersal. Evolution 23: 1-27.

Janzen, D.H. 1980. When is it coevolution? Evolution 34: 611-612.

Jordano, P. 1995. Angiosperm fleshy fruits and seed dispersers: a comparative analysis of adaptation and constraints in plant animal interactions. The American Naturalists 145: 163-191.
Kelly, C.K. \& Purvis, A. 1993. Seed size and establishment conditions in tropical trees: on the use of taxonomic relatedness in determining ecological patterns. Oecologia 94: 356-360.

Kitamura, S.; Yumoto, T.; Poonswad, P.; Chuailua, P.; Plongmai, K.; Maruhashi, T. \& Noma, N. 2002. Interactions between fleshy fruits and frugivores in a tropical seasonal forest in Thailand. Oecologia 133: 559-572.

Knighth, R.S. \& Siegfried, W.R. 1983. Inter-relationship between, type, size and colour of fruit and dispersal in Soutern African Trees. Oecologia 56: 405-412.

Leishman, M.R.; Wright, I.J.; Moles, A.T. \& Westoby, M. 2000. The evolutionary ecology of seed size. Pp. 31-57. In: M. Fenner (ed.). Seeds: the ecology of regeneration in plant communities. London, CABI Publishing.

Mack, A.L. 1998. An advantage of large seed size: tolerating rather than succumbing to seed predators. Biotropica 30: 604-608.

Mazer, S.J. \& Wheelwright, N.T. 1993. Fruit size and shape: allometry at different taxonomic levels in bird-dispersed plants. Evolutionary Ecology 7: 556-575.

Michaels, H.J.; Benner, B.; Hartgerink, A.P.; Lee, T.D.; Rice, S.; Willson, M.F.; \& Bertin, R.I. 1988. Seed size variation: magnitude, distribution, and ecological correlates. Evolutionary Ecology 2: 157-166.

Muller-Landau, H.C.; Wright, S.J.; Calderón, O.; Hubbell, S.P. \& Foster, R.B. 2002. Assessing recruitment limitation: concepts, methods and case-studies from a tropical forest. Pp. 35-54. In: D.J. Levey, W.R. Silva \& M. Galetti (eds.). Seed dispersal and frugivory: ecology, evolution and conservation. London, CABI Publishing.

Piña-Rodrigues, F.C.M.; Costa, L.G.S. \& Reis, A. 1990. Estratégias de estabelecimento de espécies arbóreas e o manejo de florestas tropicais. Pp. 676-684. In: Anais do VI Congresso Florestal Brasileiro. Campos do Jordão 1990. São Paulo, Sociedade Brasileira de Silvicultura.

Primack, R.B. 1987. Relationships among flowers, fruits, and seeds. Annual Review of Ecology and Systematics 18: 409-430.

Ridley, H.N. 1930. The dispersal of plants around the world. Kent, L. Reeve.

Rio Grande do Sul. 1997. Plano de manejo do Parque Estadual de Itapuã, RS. Porto Alegre, Departamento de Recursos Renováveis/Pró-Guaíba.

Scherer, A.; Maraschin-Silva, F. \& Baptista, L.R.M. 2007. Padrões de interações mutualísticas entre espécies arbóreas e aves frugívoras em uma comunidade de Restinga no Parque Estadual de Itapuã, RS, Brasil. Acta Botanica Brasilica 21: 203-212.

Silva, J.M.C. \& Tabarelli, M. 2000. The species impoverishment and the future flora of the Atlantic forest of northeast Brazil. Nature 404: 72-74.

Stiles, E.W. 1982. Fruit flags: two hypotheses. The American Naturalist 120: 500-509.

ter Braak, C.J.F. 1995. Ordination. Pp. 91-173. In: R.H.G. Jongman; C.J.F. ter Braak \& O.F.R. van Tongeren (eds.). Data analysis in community and landscape ecology. Cambrige, Cambrige University Press. 
Tiffney, B.H. 1984. Seed size, dispersal syndromes, and the rise of the angiosperms: evidence and hypothesis. Annals of the Missouri Botanical Garden 71: 551-576.

Tiffney, B.H. 2004. Vertebrate dispersal of seed plants through time. Annual Reviews of Ecology and Systematics 35: 1-29.

van der Pijl, L. 1982. Principles of dispersal in higher plants. New York, Springer-Verlag.

Veloso, H.P. \& Góes Filho, L. 1982. Fitogeografia brasileira: classificação fisionômica da vegetação neotropical. Boletim Técnico Projeto RADAMBRASIL, Série Vegetação 1: 1-90.

Westoby, M.; Rice, B. \& Howell, J. 1990. Seed size and plant growth form as factors in dispersal spectra. Ecology 71: 1307-1315.
Wheelwright, N.T. 1985. Fruit size, gape width, and the diets of fruit-eating birds. Ecology 66: 808-818.

Wheelwright, N.T. \& Orians G.H. 1982. Seed dispersal by animals: contrasts with pollen dispersal, problems of terminology, and constraints on coevolution. The American Naturalist 119: 402-413.

Willson, M.F.; Irvine, A.K. \& Walsh, N.G. 1989. Vertebrate dispersal syndromes in some Australian and New Zealand plant communities, with geographic comparisons. Biotropica 21: 133-147.

Willson, M.F. \& Thompson, J.N. 1982. Phenology and color in bird-dispersed fruits, or why fruits are red when they are "green". Canadian Journal of Botany 60: 701-713. 